\title{
Pulmonary sarcoidosis presenting as hoarseness and mass lesion
}

\author{
Sonam Spalgais ${ }^{1}$, Tsewang Thinles $^{2}$, Parul Mrgipuri' ${ }^{1}$, Raj Kumar ${ }^{1}$ \\ ${ }^{1}$ Department of Pulmonary Medicine, Vallabhbhai Patel Chest Institute, University of New Delhi; ${ }^{2}$ Department of \\ Otolaryngology, ESI Hospital Rohini New Delhi, India
}

\begin{abstract}
A 46-year male, ex-smoker presented with cough and hoarseness for 2-3 months. The CECT chest revealed a left upper lobe mass. BAL was negative for AFB, GeneXpert, and malignant cells. The bronchial biopsy showed granuloma with no caseation and infiltration of lymphoid cells. The ultrasound abdomen was normal. The patient was started on prednisolone $0.5 \mathrm{mg} / \mathrm{kg}$. He improved clinic-radiologically. Ex-smoker, middle-aged man with hoarseness and mass lesion on CECT chest is highly suspicious of lung malignancy. Pulmonary sarcoidosis should be kept in the differential diagnosis of unexplained hoarseness and mass lesion as the disease has diverse presentations.
\end{abstract}

Correspondence: Dr. Sonam Spalgais, Assistant Professor, Department of Pulmonary Medicine, Vallabhbhai Patel Chest Institute, University of New Delhi, New Delhi 110007, India.

Tel. +91.1127402446 .

E-mail: sosolrs@gmail.com

Contributions: All the authors made a substantive intellectual contribution. All the authors have read and approved the final version of the manuscript and agreed to be accountable for all aspects of the work.

Availability of data and materials: All data underlying the findings are fully available.

Ethics approval and consent to participate: No ethical committee approval was required for this case report by the Department, because this article does not contain any studies with human participants or animals. Informed consent was obtained from the patient included in this study.

Consent for publication: The patient gave his written consent to use his personal data for the publication of this case report and any accompanying images.

Conflict of interest: The authors declare that they have no competing interests, and all authors confirm accuracy.

Key words: Sarcoidosis; hoarseness; mass lesion.

Received for publication: 3 September 2020.

Accepted for publication: 10 November 2020.

${ }^{\circ}$ Copyright: the Author(s), 2021

Licensee PAGEPress, Italy

Monaldi Archives for Chest Disease 2021; 91:1595

doi: 10.4081/monaldi.2021.1595

This article is distributed under the terms of the Creative Commons Attribution Noncommercial License (by-nc 4.0) which permits any noncommercial use, distribution, and reproduction in any medium, provided the original author(s) and source are credited.

\section{Introduction}

Sarcoidosis is a systemic, chronic granulomatous disease of an unknown etiology that commonly affects the lungs, but all the organs can be potentially affected [1]. The diagnosis is usually done when the clinico-radiographic findings are supported by histological evidence of non-caseating granuloma and other causes of granuloma and local reactions have been reasonably excluded [13]. The common symptoms are cough, dyspnea, fatigue, weight loss, and night sweats. Approximately half of the pulmonary sarcoidosis are clinically asymptomatic [4-6]. Hoarseness is a nonspecific symptom of various benign and malignant diseases; however, it is most commonly associated with malignancy in current and ex-smokers. It is rare that, hoarseness may be the presenting symptom symptoms of pulmonary sarcoidosis [7-9]. Because of its nonspecific signs and symptoms, computed tomographic (CT) scan is the commonly used tool for confirmation of the diagnosis in sarcoidosis [2,5]. The common radiological findings include bilateral, symmetric micronodules in a peribronchovascular distribution with upper and middle lung lobes predominance accompanied by bilateral, symmetric hilar enlarged lymph nodes. However, atypical radiographic patterns of pulmonary sarcoidosis prove to be diagnostically challenging, particularly in the rare cases like mass lesions [4,10-12]. We are presenting a case of pulmonary sarcoidosis with high suspicion of lung malignancy, who was presented with hoarseness for 3 month and mass lesion on chest CECT scan.

\section{Case Report}

A 46-year-old male presented with complaints of cough for 3 months and hoarseness for 2 months. He had no history of chest pain, wheeze, fever, loss of weight and loss of appetite. He was an ex-smoker with 10 pack years. His general physical and respiratory examination was normal. There was no peripheral palpable lymph node. Otorhinolaryngologist evaluation for hoarseness was unremarkable. His routine blood investigation did not showed any abnormality. Chest X-ray (PA view) showed left hilar opacity. The sputum for direct acid fast bacilli (AFB) staining, liquid culture for M. tuberculosis (M. Tb), GeneXpert and malignant cell was negative. Serum angiotensin converting enzyme was $34 \mathrm{IU} / \mathrm{ml}$ and other connective tissue serology were also negative. Pulmonary function test showed normal value of spirometry and diffusion capacity of carbon monoxide (DLCO). The contrast enhanced computer tomography (CECT) chest revealed mass lesion $(3.9 \times 3.0 \times 3.7 \mathrm{~cm})$ in aortopulmonary window of left upper mediastinal region encircling and stenosing the upper lobe bronchioles and pulmonary vessels and subcentime- 
ter mediastinal lymphnodes (Figure 1). On video-bronchoscopy vocal cord was normal. Carina was sharp. All the opening of both sides was normal with few nodules were seen in left main and upper lobe bronchus. Bronchioalveolar lavage (BAL) was negative for AFB, GeneXpert and malignant cell. The bronchial biopsy from left upper lobe showed poorly formed granuloma with no evidence of caseation and moderate infiltration of lymphoid cells, consistent with sarcoidosis (Figure 2). BAL fluid culture for $M$. $T b$ and fungus was also negative. The ultrasound abdomen and ophthalmological examination were also normal. Due to high clinical suspicion of malignancy, patient was advised repeat bronchoscopic biopsy to confirm diagnosis. Patient refused for further investigation. Thus, patient was started on steroid therapy (prednisolone) at the dose of $0.5 \mathrm{mg} / \mathrm{kg}$ with the provisional diagnosis of sarcoidosis followed by tapering of $5 \mathrm{mg} 2$ weekly with regular follow up. His cough and hoarseness improved significantly within 2 months of treatment. The CECT chest after 3 months of treatment also showed significant decrease in size of mass lesion $(2.2 \times 3.0 \times 2.2 \mathrm{~cm})$ (Figure 3$)$. The dose of prednisolone was tapered further 2 weekly till $5 \mathrm{mg}$ once daily. He was kept on $5 \mathrm{mg}$ daily dose for 3 months and $5 \mathrm{mg}$ alternate day for the next 3 months. His CECT chest after one year of treatment showed decrease in size of mass lesion. The symptoms were completely resolved, and treatment was stopped. He is on regular follow up for 6 months with no symptoms.

\section{Discussion}

The usual symptoms of sarcoidosis are cough, dyspnea, fatigue, weight loss, and night sweats [4-6]. Hoarseness can be the presenting symptom of various benign and malignant diseases. However hoarseness of 2 month in ex-smoker with mass lesion is usually a symptom of malignancy. There are few reported cases of pulmonary sarcoidosis with hoarseness as presenting symptom. The mechanisms for hoarseness in sarcoidosis may be explained by: i) compression of the left recurrent laryngeal nerve by enlarged mediastinal lymph node; Enlargement of mediastinal lymph node is one of manifestation of sarcoidosis particularly hilar lymph node; The left recurrent laryngeal nerve has almost double the length of the right. Because of its longer course, it is more susceptible to be compressed, which leads to hoarseness; the common site of recurrent laryngeal nerve compression is at the level of the arch of the aorta [7-9]. ii) Laryngeal infiltration by sarcoid granuloma with normal vocal cord motility; this is seen in nearly $5 \%$ of cases $[8,13]$. iii) Cranial polyneuropathy in sarcoidosis may lead to hoarseness and dysphagia by affecting vagus nerve, such condition is commonly associated with other neuropathies particularly facial nerve palsy [7]. In our case the hoarseness may be due to first 2 reasons.

Because of non-specific clinical presentation and no definitive diagnostic test for sarcoidosis, CECT chest is commonly used tool

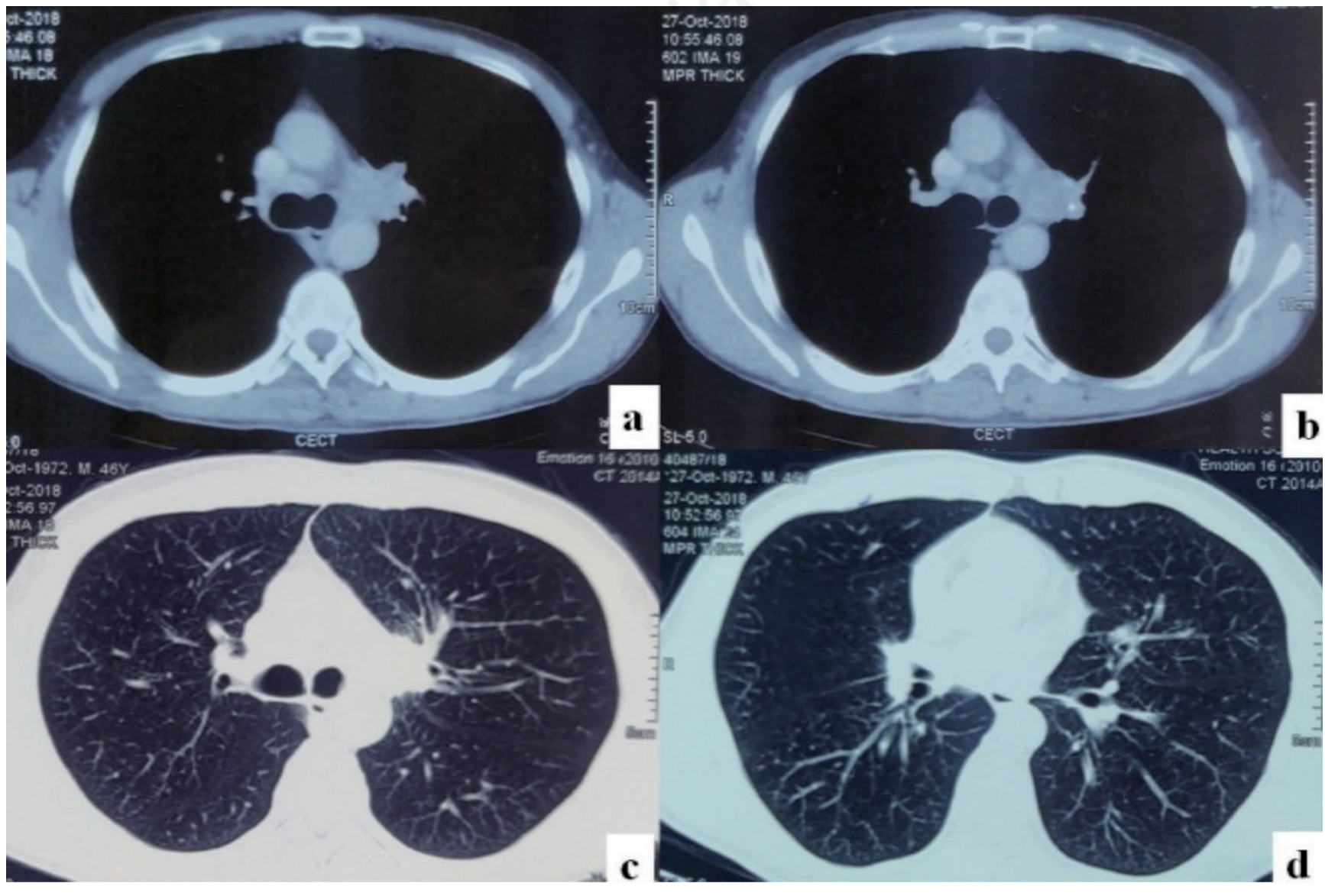

Figure 1. CECT chest at presentation. a,b) CECT chest mediastinal window showed mass lesion $(3.9 \times 3.0 \times 3.7 \mathrm{~cm})$ with calcification in aortopulmonary window of left upper mediastinal region. c, d) Lung window showed normal lung parenchyma. 


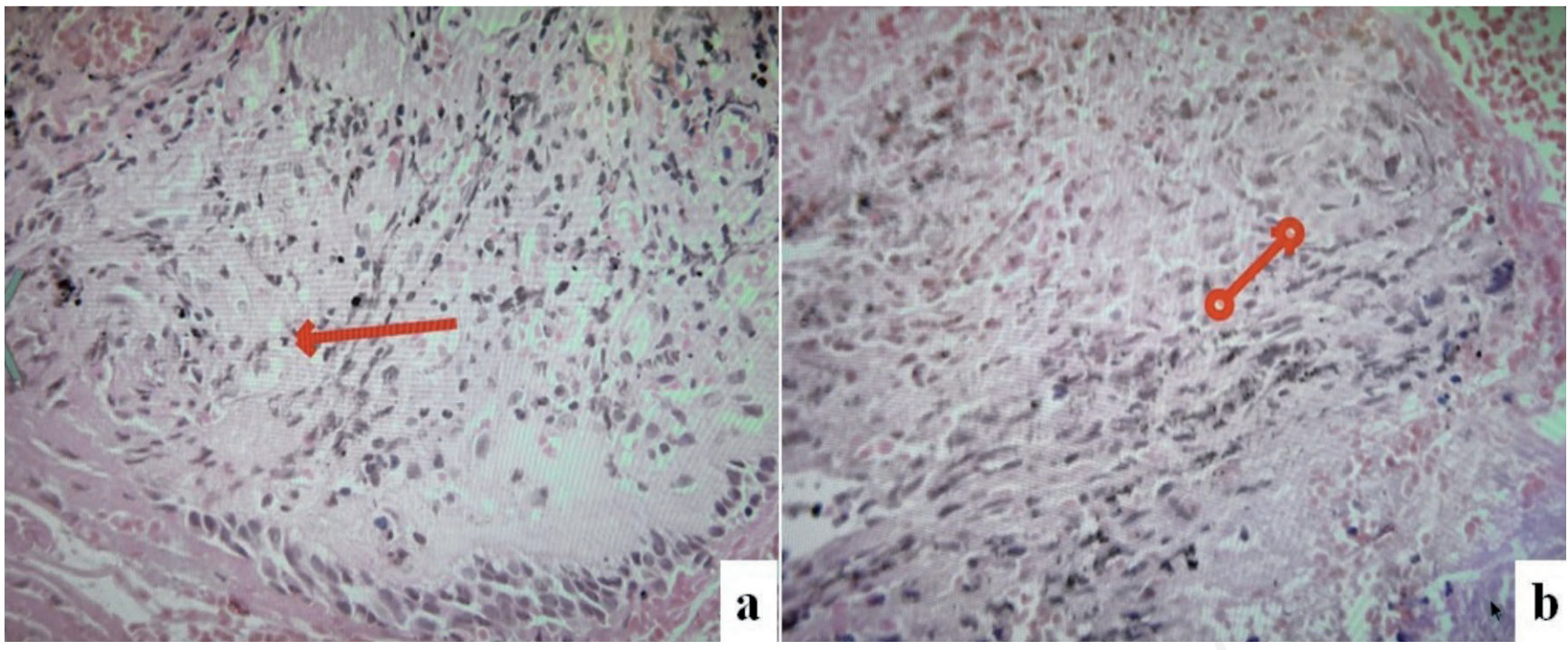

Figure 2. Histopathology of endobronchial biopsy showed poorly form granuloma with no evidence of caseation and moderate infiltration of lymphoid cells.

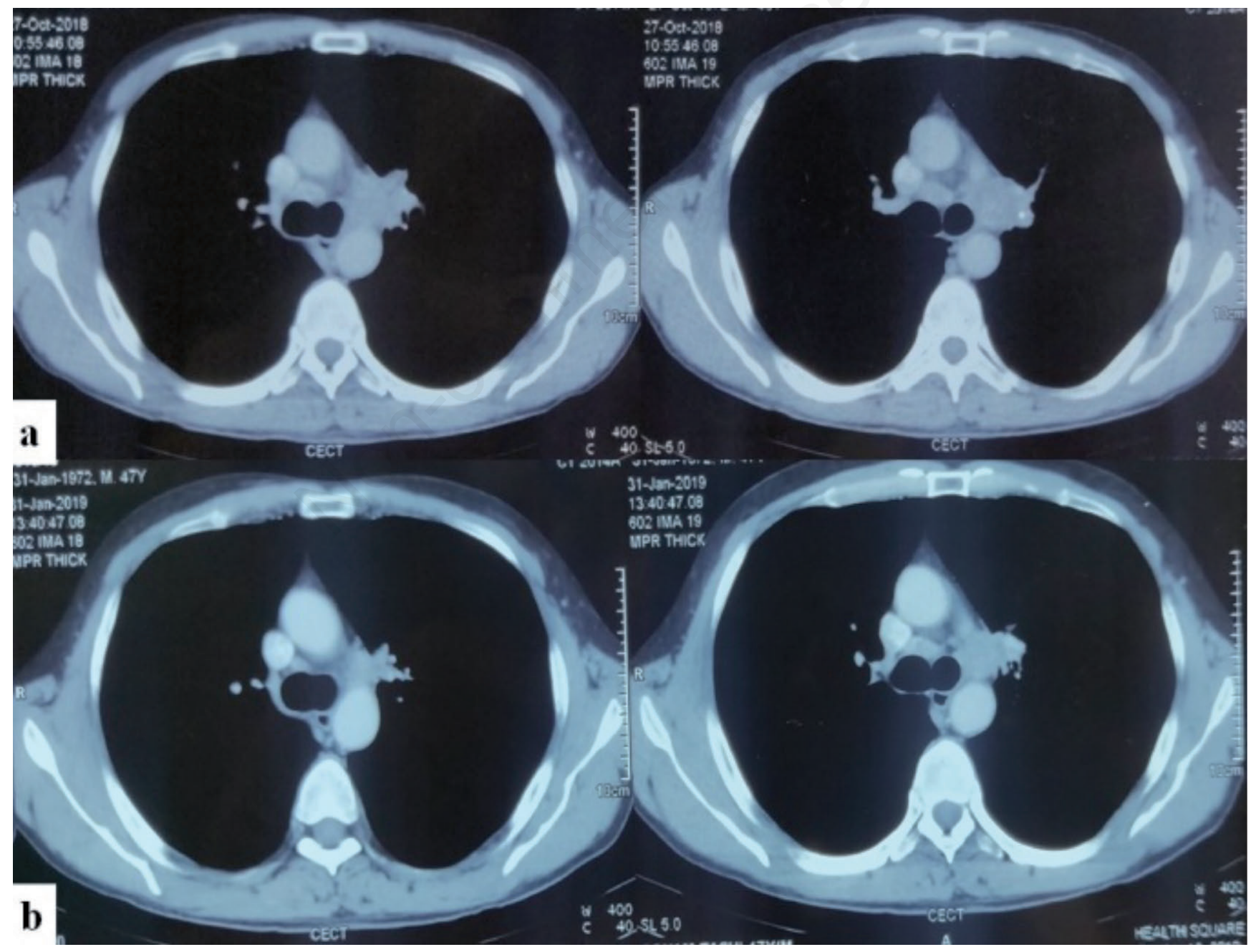

Figure 3. a) CECT chest at presentation showed mass lesion of $3.9 \times 3.0 \times 3.7 \mathrm{~cm}$. b) Decrease in size of lesion to $2.2 \times 3.0 \times 2.2 \mathrm{~cm}$ after 3 month treatment. 
for confirmation. The nonspecific or atypical radiological findings are seen in $25 \%-30 \%$ of patients [1,2,4]. However, a lung mass, mediastinal lymph nodes without hilar lymphadenopathy and lymph node calcification are rare presentation of sarcoidosis $[4,11,12]$. Pulmonary sarcoidosis presenting as lung mass on CT chest is a very rare manifestation limited to case reports $[11,12]$. As in our case pulmonary sarcoidosis with lung mass on CT chest with hoarseness in ex-smoker is highly suspicious of malignancy. The presence of granulomatous inflammation in biopsy did not confirm the diagnosis as the granuloma is seen in some small cell lung cancer and sarcoid-like reaction are seen in or near the malignancy or regional draining lymph nodes $[11,12]$. To confirm diagnosis of sarcoidosis with an atypical presentation, biopsy from two noncontiguous sites is recommended $[11,12,14]$. However, it was not possible to biopsy from other site in our case as there was no evidence of extrapulmonary involvement. Also our patient refused for repeat bronchial biopsy. So, he was treated with oral corticosteroid (prednisolone) and kept on regular follow up. Therapeutic trial with corticosteroid treatment in atypical presentation of sarcoidosis is also one of the diagnostic options [14]. He responded well clinically within 2 months and for confirmation we did his repeat CECT chest after 3 months, which also revealed significant decrease in size of the mass. There are cases of pulmonary sarcoidosis presenting as lung mass or hoarseness in literature. Our case is unique form of sarcoidosis presenting with both lung mass and hoarseness.

\section{Conclusions}

It is well known that pulmonary sarcoidosis is difficult to differentiate from lung malignancy and other granulomatous diseases. We report a case of sarcoidosis with a high clinical suspicion of lung malignancy. Lung mass on CT chest with hoarseness in exsmoker is a very unique presentation of sarcoidosis.

\section{References}

1. Hunninghake GW, Costabel U, Ando M, et al. American Thoracic Society/European Respiratory Society/World Association of Sarcoidosis and other Granulomatous Disorders. SarcoidosisVasc Diffuse Lung Dis 1999;16:149-73.

2. Baughman RP, Culver DA, Judson MA. A concise review of pulmonary sarcoidosis. Am J Respir Crit Care Med 2011;183:573-81.

3. Judson MA. The diagnosis of sarcoidosis attempting to apply rigor to arbitrary and circular reasoning. Chest 2018;154:1006-7.

4. Criado E, Sánchez M, Ramírez J, et al. Pulmonary sarcoidosis: Typical and atypical manifestations at high- resolution CT with pathologic correlation. Radiographics 2010;30:1567-86.

5. Iannuzzi MC, Rybicki BA, Teirstein AS. Sarcoidosis. N Engl J Med 2007;357:2153-65.

6. Baughman RP, Teirstein AS, Judson MA, et al. Clinical characteristics of patients in a case control study of sarcoidosis. Am J Respir Crit Care Med 2001;164:1885-9.

7. Lim K H, Liam CK and Wong CM. A patient presenting with hoarseness. Postgrad Med J 2000;76:510-9.

8. El-Kassimi FA, Ashour M, Vijayaraghavan R. Sarcoidosis presenting as recurrent left laryngeal nerve palsy. Thorax 1990;45:565-6.

9. Mastan S, Advani R, Stobbs N, et al A rare manifestation of a multisystemic disease: a case of vocal cord palsy secondary to sarcoidosis Case Reports 2015;2015:bcr2015209728.

10. Honda O, Johkoh T, Ichikado K, et al. Comparison of high resolution CT findings of sarcoidosis, lymphoma, and lymphangitic carcinoma: Is there any difference of involved interstitium? J Comput Assist Tomogr 1999;23:374-9.

11. Margaritopoulos GA, Proklou A, Lagoudaki E, et al. Sarcoidosis in a 65 -year-old woman presenting with a lung mass and pericardial effusion: a case report. J Med Case Rep 2012;6:259.

12. Kelleher DW, Homer R, Herzog EL, Ryu C. A rare presentation of pulmonary sarcoidosis as a solitary lung mass: a case report. J Med Case Rep 2018;12:94.

13. Neville E. Upper respiratory tract sarcoidosis. Semin Respir Med 1986;8:52-8

14. Moller DR. Rare manifestations of sarcoidosis. ERS Monograph 2005;32:233-50. 